\title{
Thomson parabola and time-of-flight detector cross-calibration methodology on the ALLS 100 TW laser-driven ion acceleration beamline
}

Cite as: Rev. Sci. Instrum. 91, 103303 (2020); https://doi.org/10.1063/5.0020257

Submitted: 29 June 2020 • Accepted: 02 October 2020 • Published Online: 16 October 2020

(D) S. Vallières, M. Salvadori, P. Puyuelo-Valdes, et al.

\section{ARTICLES YOU MAY BE INTERESTED IN}

Design and optimization of a proportional counter for the absolute determination of lowenergy x-ray emission rates

Review of Scientific Instruments 91, 103304 (2020); https://doi.org/10.1063/5.0013909

Real-time signal processing in field programmable gate array based digital gamma-ray spectrometer

Review of Scientific Instruments 91, 104707 (2020); https://doi.org/10.1063/5.0005694

A new method for high-bandwidth servo control of the power ratio between two Raman beams for cold atom interferometer

Review of Scientific Instruments 91, 103109 (2020); https://doi.org/10.1063/5.0020144

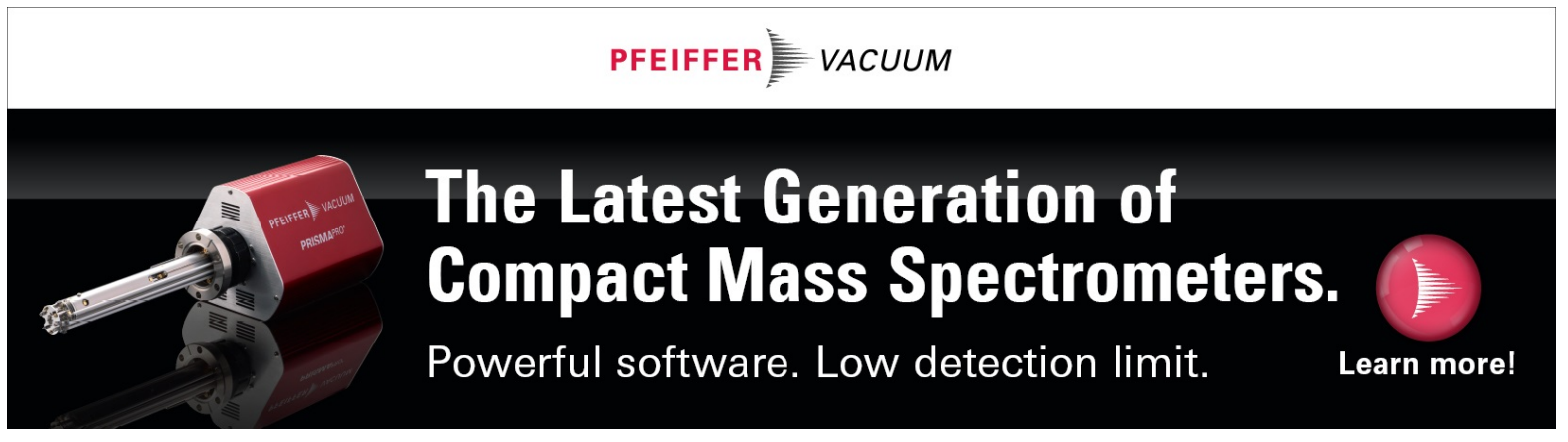




\title{
Thomson parabola and time-of-flight detector cross-calibration methodology on the ALLS 100 TW laser-driven ion acceleration beamline
}

Cite as: Rev. Sci. Instrum. 91, 103303 (2020); doi: 10.1063/5.0020257

Submitted: 29 June 2020 - Accepted: 2 October 2020 •

Published Online: 16 October 2020

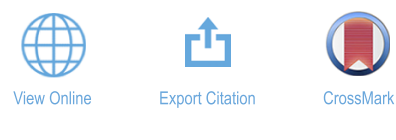

S. Vallières, ${ }^{1,2, a)}$ (D) M. Salvadori, ${ }^{1,3,4}$ P. Puyuelo-Valdes, ${ }^{1,5}$ S. Payeur, ${ }^{1}$ S. Fourmaux, ${ }^{1}$ (D) F. Consoli, ${ }^{3}$ (D) C. Verona,

E. d'Humières, ${ }^{2}$ M. Chicoine, ${ }^{7}$ S. Roorda, ${ }^{7}$ F. Schiettekatte, ${ }^{7}$ and P. Antici'

\author{
AFFILIATIONS \\ ${ }^{1}$ INRS-EMT, 1650 Boul. Lionel-Boulet, Varennes, Quebec J3X 1P7, Canada \\ ${ }^{2}$ CELIA, Univ. of Bordeaux, 351 Cours de la Libération, Talence 33400, France \\ ${ }^{3}$ ENEA, Via Enrico Fermi 45, Frascati 00044, Italy \\ "Univ. of Rome "La Sapienza", P. Aldo Moro 5, Roma 00185, Italy \\ ${ }^{5}$ CENBG, CNRS-IN2P3, 19 rue du Solarium, Gradignan 33175, France \\ “Univ. of Rome "Tor Vergata", Via Cracovia 50, Roma 00133, Italy \\ ${ }^{7}$ Univ. of Montréal, 2900 Boul. Édouard-Montpetit, Montréal, Quebec H3T 1J4, Canada
}

${ }^{a)}$ Author to whom correspondence should be addressed: simon.vallieres@emt.inrs.ca

\begin{abstract}
We report on the cross-calibration of Thomson Parabola (TP) and Time-of-Flight (TOF) detectors as particle diagnostics, implemented on the most recent setup of the ALLS 100 TW laser-driven ion acceleration beamline. The Microchannel Plate (MCP) used for particle detection in the TP spectrometer has been calibrated in intensity on the tandem linear accelerator at the Université de Montréal. The experimental data points of the scaling factor were obtained by performing a pixel cluster analysis of single proton impacts on the MCP. A semi-empirical model was extrapolated and fitted to the data to apply the calibration also to higher kinetic energies and to extend it to other ion species. Two TOF lines using diamond detectors, placed at $+6^{\circ}$ and $-9^{\circ}$ with respect to the target-normal axis, were benchmarked against the TP spectrometer measurements to determine the field integrals related to its electric and magnetic dispersions. The mean integral proton numbers obtained on the beamline were about $4.1 \times 10^{11}$ protons/sr with a standard deviation of $15 \%$ in the central section of the spectrum around $3 \mathrm{MeV}$, hence witnessing the high repeatability of the proton bunch generation. The mean maximum energy was of $7.3 \pm 0.5 \mathrm{MeV}$, well in agreement with similar other $100 \mathrm{TW}$-scale laser facilities, with the best shots reaching $9 \mathrm{MeV}$ and nearly $10^{12}$ protons/sr. The used particle diagnostics are compatible with the development of a high-repetition rate targetry due to their fast online readout and are therefore a crucial step in the automation of any beamline.
\end{abstract}

Published under license by AIP Publishing. https://doi.org/10.1063/5.0020257

\section{INTRODUCTION}

The precise measurement of particle characteristics is of the utmost importance when it comes to using particle beams for different applications. The development of particle diagnostics heavily depends on its features, e.g., the energy range to be detected, the bunch charge and particle charge state, the intensity of the beam, the beam profile, the required sensibility, and the resolution. For these reasons, a plethora of different diagnostics have been developed and tested in the past for measuring particle beams, taking into consideration the multiple criteria that they need to satisfy. ${ }^{1}$ High-intensity, short-pulse laser-driven proton beams, discovered nearly two decades ago, ${ }^{2}$ and today routinely accelerated with the so-called Target-Normal Sheath Acceleration (TNSA) mechanism, ${ }^{3}$ have triggered the development of new diagnostics. These laserdriven particle beams have the following advantages: they are very short in duration (ps at the source) ${ }^{4}$ and high in current (kA), with a large energy spread and low emittance; they can be operated at 
$\mathrm{Hz}$ repetition rates; and they can generate strong Electromagnetic Pulses (EMPs) during their production. ${ }^{8}$ This requires detectors that are highly versatile since they need to allow for a quick acquisition time, high dynamic range, wide-range energy response, quick online readout, and high resistance to EMPs. Combining all these requirements is challenging and not always possible using detectors typically employed in the conventional accelerator community.

Numerous detectors have been developed and tested in the last few years to measure laser-accelerated protons. Among the most used detectors are stacks of Radiochromic Films (RCFs), which are polyester sheets with a sensitive layer that darken upon exposure to ionizing radiation. They allow for a discrete spectrum and $2 \mathrm{D}$ beam profile reconstruction but are not suitable for high-repetition rate detection since their readout requires the use of a scanner as a subsequent step after the irradiation to post-process the data. They are also not sensitive to low particle intensities and to low proton energies $(<1 \mathrm{MeV})$, energy regions where the proton number is significant for laser-driven sources. A faster and more sensitive readout is performed by replacing RCFs with scintillators. Thomson Parabolas (TPs) allow for an almost continuous spectrum retrieval in one dimension. The detection of the protons is performed using image plates, semiconductor-based detectors, or Microchannel Plates (MCPs). The latter two have the advantage of being usable with higher-repetition rates and have therefore been employed in several experiments where a quick analysis was needed (see review articles on TNSA experiments ${ }^{2,10,11}$ and references therein). The Time-of-Flight (TOF) technique ${ }^{12,13}$ is another well-established method used to retrieve information about the spectrum of the accelerated particles. Recently, TOF delay lines were used employing Chemical Vapor Deposition Diamond Detectors (CVD-DDs). ${ }^{14}$ These detectors are characterized by a fast temporal response compared to scintillator-based detectors, ${ }^{15}$ provide a high-energy resolution, and are optimized to work in environments highly polluted by EMPs. The compactness of these detectors allows us to place several TOF lines at different angles, providing a simultaneous measurement of the accelerated particle beam even within a rather small cone of $20^{\circ}$ half-angle, i.e., the typical beam aperture of laser-accelerated proton beams. TOF detectors can be employed simultaneously with a Thomson parabola, which, on the other hand, provides information about the ion species but generally is more cumbersome and has a lower energy resolution than TOF. A detector based on acoustic waves (I-BEAT) has been lately proposed to overcome EMP problems and enable high-repetition rate online reconstruction of the absolute depth-dose distribution at application sites. ${ }^{16}$ However, this diagnostic has not yet been extensively used by other groups. Among all the above cited diagnostics, MCP-TP and TOF coupled with diamond detectors (DD-TOF) are consolidated as being the most promising and appropriate route to diagnose intense proton beams as obtained on commercially available high-repetition rate laser-systems.

In this paper, we present a method to calibrate MCPs used with TP and we use two different diamond detectors employed as TOF detectors. The MCP used for the particle detection with the TP spectrometer has been calibrated in intensity on the $2 \times 6 \mathrm{MV}$ tandem linear accelerator at the Université de Montréal, to retrieve the particle scaling factor that is needed to determine the particle numbers observed in the MCP-TP spectrometer for protons and carbon ions. We then perform a cross-calibration between the different diagnostics in a real-setting scenario, highlighting benefits and drawbacks: using the maximum proton energies obtained from the CVD$\mathrm{DD}$, we calibrate the relevant parameters of the TP spectrometer required for the kinetic energy retrieval, and using the calibration of the proton numbers obtained on the tandem accelerator, we validate the calibration of the CVD-DD. The detectors are used to optimize the proton beam generation on the recently installed ALLS 100 TW laser-driven ion acceleration beamline. This paper is organized as follows: we first present the methodology for calibrating the response of a MCP using a pixel cluster analysis. The results of the MCP calibration allow us to establish a semi-empirical modeling of the response function, applicable for similar MCP types. In Sec. II, we present a description of the setup on the laser-driven ion acceleration beamline, along with a cross-calibration methodology for the calibration of the field integrals to determine the particle's kinetic energy with the TP spectrometer. We describe the implementation of the diagnostics on the Advanced Laser Light Source (ALLS) 100 TW laser-driven ion beamline and its use for maximizing the ion yield.

\section{ABSOLUTE MCP RESPONSE CALIBRATION}

In this section, we detail how to relate the absolute particle number to the intensity of an MCP image as read by the CCD camera, through the measurement of the kinetic energy-dependent scaling factor SF for ions.

\section{A. MCP detection system}

We consider the MCP detection system as the interplay of the image acquisition system coupled to the MCP-phosphor assembly. In our setup, we used a double MCP detector in the chevron configuration with $97 \times 79 \mathrm{~mm}^{2}$ sensitive area and $8^{\circ}$ bias angle pores of $25 \mu \mathrm{m}$ diameter with 1:40 aspect ratio, providing very high gain $\left(10^{6}-10^{7}\right)$ suitable for particle counting, acquired from the company Photonis. ${ }^{17}$ The chevron MCP is assembled with a P43 phosphor, well known for its high light yield but a slow decay time of $3.2 \mathrm{~ms}$ at $1 \%$ of maximum intensity. Nevertheless, the detector refreshment time is far more than sufficient for the present study and allows for potential repetition rates of hundreds of $\mathrm{Hz}$. The MCP was imaged with an $f / 2.4$ objective lens placed at $52 \mathrm{~cm}$ behind the phosphor, mounted on a 1.3 megapixel Blackfly PoE GigE CCD camera from $F L I R,{ }^{18}$ using a 12-bit dynamic range. A potential difference of $-1.5 \mathrm{kV}$ was applied between the entrance (cathode) and the exit (anode) of the MCP to drive the electron avalanche. The output electrons were then accelerated by a $+5 \mathrm{kV}$ potential placed on the phosphor screen for additional gain through light conversion.

\section{B. Calibration on tandem accelerator}

To calibrate the MCP detection system, different proton irradiations were performed on the $2 \times 6 \mathrm{MV}$ tandem linear accelerator at the Université de Montréal, using incident proton kinetic energies of $\mathcal{E}_{\mathrm{K}, 0}=1 \mathrm{MeV}, 2.5 \mathrm{MeV}, 5 \mathrm{MeV}, 7.5 \mathrm{MeV}, 10 \mathrm{MeV}$, and $11.5 \mathrm{MeV}$. A $2 \times 2 \mathrm{~mm}^{2}$ collimated square transverse profile, non-divergent, and monoenergetic proton beam was incident on a $1 \mathrm{~cm}^{2}, 100 \mathrm{~nm}$ thick gold thin foil used as a Rutherford scatterer, placed at $45^{\circ}$ with 
respect to proton incidence. Due to the used kinetic energies and the very small thickness of the foil, the vast majority of incident protons was transmitted through the Au foil and was collected with a Faraday Cup (FC) calibrated in absolute terms for charge monitoring. We used transmitted currents of $\dot{Q}=0.1 \mathrm{nA}, 1 \mathrm{nA}$, and 10 $\mathrm{nA}$ for each chosen kinetic energy. The transmitted currents were compared to current measurements without a gold foil to determine the proton current that was incident on the foil during the acquisitions. This technique allows us to compensate in situ for the strongly varying scattering cross section with kinetic energy within the range of $1 \mathrm{MeV}-11.5 \mathrm{MeV}$ and ensured that we had sufficient scattering events collected by using the detector. A voltage bias of $+200 \mathrm{~V}$ was applied on the foil to collect electrons ejected due to physical processes. All irradiations were performed in the dark and under high vacuum $\left(10^{-7} \mathrm{mbar}\right)$ with the camera acquisition time set to $400 \mathrm{~ms}$. Acquisitions were repeated $N=10$ times for each irradiation type to ensure significant particle counting statistics. Hence, for each camera acquisition, incident proton fluences on the gold foil varied in the range of $\phi=(0.7-21.2) \times 10^{11}$ protons $/ \mathrm{cm}^{2}$. The scattered protons impacted the MCP's surface, located perpendicular to the proton incidence direction at a distance of $88 \mathrm{~cm}$ from the gold foil, over a $5 \times 4 \mathrm{~cm}^{2}$ area, giving a particle collection solid angle of $2.6 \mathrm{msr}$. We performed scattering event simulations with the software SIM$N R A,{ }^{19}$ a Rutherford Backscattering Spectroscopy (RBS) calculation tool used for RBS analysis. This helped to ensure proper matching between simulated and detected scattered proton numbers, as well as to retrieve the kinetic energies of the protons scattered on the MCP, which are used for further analysis. The number of scattered protons hitting the MCP per camera acquisition varied from a few tens to a few hundreds counts depending on the used kinetic energy, hence allowing us to discriminate single particle events over the $20 \mathrm{~cm}^{2}$ detection surface. A schematic diagram of the experimental setup is shown in Fig. 1.

\section{Pixel clustering analysis}

In order to analyze the intensities of the pixel clusters on the image recording the MCP irradiated using monoenergetic protons, as shown in the inset of Fig. 1, a numerical method was developed based on the work of Baumann et al. ${ }^{20}$ Since the number of scattered protons hitting the detector is on the order of a few tens to a few hundreds of protons per camera acquisition, the probability of having two protons hitting the same microchannel within this timeframe is sufficiently low (probability $<5 \times 10^{-4}$ ) such that we can reasonably assume that the pixel clusters observed on the acquisition image are cones of light emitted by the phosphor from single proton events. The spatial resolution for this kind of MCP detection system is limited by the Point Spread Function (PSF) of phosphor, which generates cones of light spatially larger than the diameter of a microchannel $(25 \mu \mathrm{m})$ and of the pixel size of the image acquisition system, which, using a lens magnification, relays a surface size on the MCP of $90 \mu \mathrm{m}$ onto a single CCD pixel. In the x-ray photon counting method using pixelated semiconductor $\mathrm{x}$-ray detectors, it is possible that one $\mathrm{x}$-ray photon produces a single pixel event with no charge leakage to the surrounding pixels. Here, it is necessary to analyze the pixel intensity distribution for each cluster produced by independent protons. Therefore, this issue prevents us from using

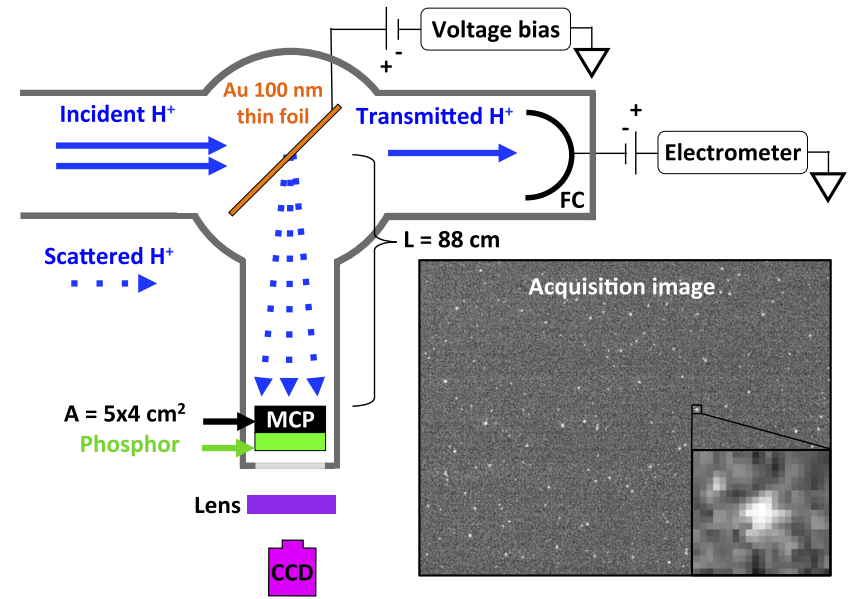

FIG. 1. Experimental setup for measuring scattered proton irradiations on the tandem particle accelerator at the Université de Montréal. A $2 \times 2 \mathrm{~mm}^{2}$ collimated square transverse profile, non-divergent, and monoenergetic proton beam is incident on a gold thin foil acting as a Rutherford scatterer. Most protons are transmitted through the gold foil and are collected with a Faraday Cup for fluence monitoring, whereas a fraction of the scattered protons are incident on the MCP detection system for calibration. The inset is an acquisition image of phosphor recorded with the CCD camera.

single pixel events for histogram reconstruction. The intensity distributions were evaluated in the filtered domain using the following equation:

$$
I_{\text {filt }}(i, j)=\left[I(i, j)-I_{\text {blank }}(i, j)\right] \star \frac{1}{2 \pi} e^{-\frac{i^{2}+j^{2}}{2}}>T_{\text {noise }},
$$

where $I(i, j)$ is the raw acquisition image evaluated at the pixel coordinates $(i, j), I_{\text {blank }}(i, j)$ is a blank image acquired without proton current (i.e., $\dot{Q}=0$ ), « denotes the cross-correlation operator, and $T_{\text {noise }}$ is an intensity threshold used for background suppression. A $2 \mathrm{D}$ cross correlation with a normalized Gaussian kernel with a unitary standard deviation is performed as a noise reduction technique and to enhance the cluster intensities with respect to the background, hence increasing the signal-to-noise ratio (SNR) and thus facilitating the search for the local maximum at each pixel cluster. The intensity threshold $T_{\text {noise }}$ is defined empirically as the minimum intensity value such that $I_{\text {filt }}(i, j)$ encompasses only the pixel clusters coming from proton hits and is kept constant for all images throughout the analysis. The retrieval of the mean pixel intensity is then based on the clustering method described by Baumann et al. ${ }^{20}$ The method consists in considering the first neighbors around the maximum pixel position within a cluster (i.e., a $3 \times 3$ array) and then taking the average value of the pixels above $T_{\text {noise }}$. This method, as opposed to other techniques such as 4 -Pix (i.e., $2 \times 2$ array), cross shape, or nearest neighbors also shown by Baumann et al., ${ }^{20}$ allows us to consider a general shape that the pixel cluster might have, hence not limiting the search to a particular cluster shape that could induce a misestimation of the mean intensity value. The proton scaling factor $\mathrm{SF}_{\text {protons }}$ is obtained as follows: We produce histograms with the cluster intensity ( $\mathrm{x}$ axis) and its occurrence (y axis). We cumulate this process over $N=10$ images to ensure sufficient particle counting statistics. $\mathrm{SF}_{\text {protons }}$ is calculated by taking the mean 
cluster intensity of the distribution ( $\mathrm{x}$ axis). The total standard deviation of $\mathrm{SF}_{\text {protons }}, \sigma_{\mathrm{SF}}$, obtained by independent observations from each image acquisition is given by the following equation:

$$
\sigma_{\mathrm{SF}}=\sqrt{\left[\sum_{n=1}^{n=N}\left(\frac{1}{\sigma_{n}^{2}}\right)\right]^{-1}} .
$$

\section{Experimental results}

In this section, we detail the experimental results obtained on the tandem accelerator and the semi-empirical modeling of the scaling factor (SF) function. Based on several previous works, ${ }^{21-25}$ we have modeled the intensity response of the MCP, $\mathcal{R}_{\mathrm{MCP}}$, for $\mathrm{MeV}$-ranged ions with the following equation, as proposed by Prasad et al. ${ }^{26}$ and Jeong et al.:

$$
\mathcal{R}_{\mathrm{MCP}}\left(\mathcal{E}_{\mathrm{K}, 0}\right) \propto \int_{0}^{l}\left[\frac{d \mathcal{E}_{\mathrm{K}}}{d z}\right]_{\mathrm{e}}\left(\mathcal{E}_{\mathrm{K}, 0}, z\right) e^{-\frac{z}{l_{g}}} \mathrm{~d} z .
$$

In Eq. (3), $\left[\frac{d \mathcal{E}_{\mathrm{K}}}{d z}\right]_{\mathrm{e}}$ is the electronic stopping power for a particular ion species in a given material, $e^{-\frac{z}{l_{g}}}$ models the MCP's exponential gain variation with microchannel penetration depth $z$, $l_{\mathrm{g}}$ is the characteristic gain length, $l$ is the microchannel length, and $\mathcal{E}_{\mathrm{K}, 0}$ is the initial kinetic energy of the particle incident on the detector. Indeed, an energy deposition through ion impact ionization at the entrance of a microchannel will generate a stronger electron avalanche than farther in the channel, justifying the choice of the exponential gain weight. The output response is thus modeled as the sum over the entire channel length of all incremental gain-weighted energy depositions as a function of depth. The amplitude scaling of the gain from the MCP detection system is then obtained by a linear fitting to scale the MCP response function $\mathcal{R}_{\mathrm{MCP}}$ to the particle scaling factor (SF) as measured by CCD camera counts per incident particle, estimated by the following equation:

$$
\mathrm{SF}=a \cdot \mathcal{R}_{\mathrm{MCP}}+b .
$$

We note that $a, b$, and $l_{\mathrm{g}}$ are characterized merely by the MCP detection system and are, therefore, particle-independent, whereas the particle dependence is solely considered in the electronic stopping power. For the modeling of experimental data, we have used the proton electronic stopping power in leaded-glass, which is the main material of the microchannels, as computed by the software SRIM. ${ }^{28}$ Using the experimental data points for the proton scaling factor $\mathrm{SF}_{\text {protons }}$, we have iteratively varied $l_{\mathrm{g}}$ and subsequently fitted $a$ and $b$, until best match was achieved by minimizing the Sum of Squared Errors (SSEs) as a cost function over the sampled kinetic energy data points. The results of this best match modeling are shown in Fig. 2. At first, it is possible to observe, in Fig. 2(a), the numerically generated Bragg peaks for three incident proton energies of $1 \mathrm{MeV}$, $2.5 \mathrm{MeV}$, and $5 \mathrm{MeV}$, overlaid by a plot of the exponential gain weight $e^{-\frac{z}{l_{g}}}$ that provides the best matching of the model with the experimental data [see the right scale in Fig. 2(a)]. We find a fit optimum for $l_{\mathrm{g}}=11.39 \mu \mathrm{m}$, along with the linear scaling parameters of $a=221.7$ counts/particle $/ \mathrm{MeV}$ and $b=563.6$ counts/particle. For low-energy protons, for instance, $1 \mathrm{MeV}$ [red full curve in Fig. 2(a)],
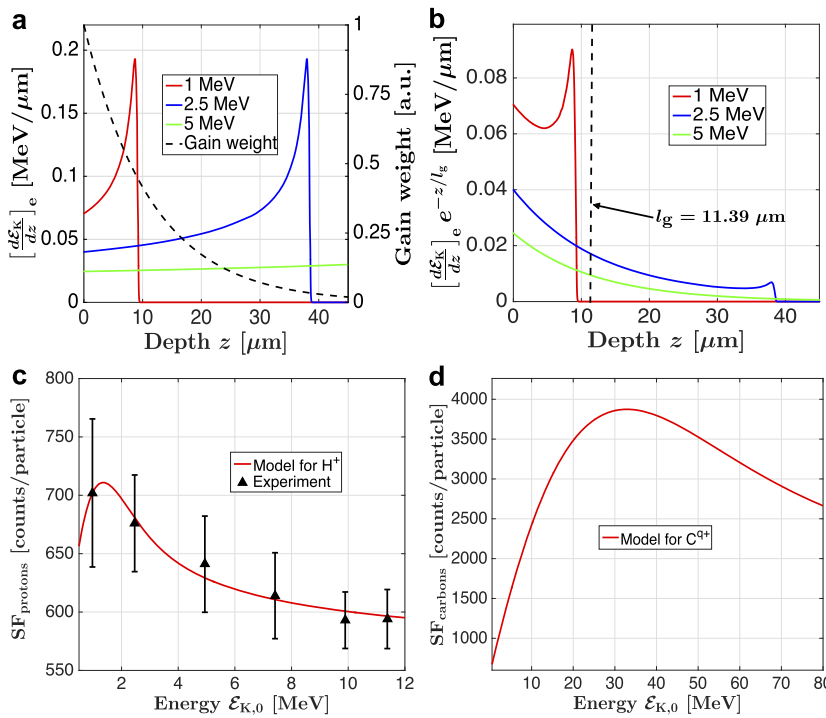

FIG. 2. MCP detection system experimental response and semi-empirical modeling. (a) Proton electronic stopping power in leaded-glass as a function of depth (Bragg peak) for different initial kinetic energies of $\mathcal{E}_{\mathrm{K}, 0}=1$ (red full curve), 2.5 (blue full curve), and $5 \mathrm{MeV}$ (green full curve), along with the best-matching exponential gain weight as a function of depth $x$ (black dashed curve), obtained for $I_{\mathrm{g}}=11.39 \mu \mathrm{m}$. (b) Gain-weighted electronic stopping power variation with depth. (c) Experimental proton scaling factor $\mathrm{SF}_{\text {protons (black triangles) and best fit model }}$ (red full curve), with $R^{2}=0.976$. In the presented case, the best fit is obtained for $a=221.7$ counts/particle/MeV and $b=563.6$ counts/particle. (d) Extrapolation of the best fit model for carbon ions.

the entire energy deposition of the particle occurs at the front surface of the MCP within the first few micrometers, where the gain is highest. As the incident kinetic energy increases, for instance, with $2.5 \mathrm{MeV}$ and $5 \mathrm{MeV}$ protons [blue and green full curves in Fig. 2(a), respectively], the position of the Bragg peak shifts to after the high gain zone, where we consider the region located before $l_{\mathrm{g}}$ as the high gain zone. This decreases the gain-weighted energy deposition variation with depth, as shown in Fig. 2(b). The integral of the curves presented in Fig. 2(b) for different kinetic energies $\mathcal{E}_{\mathrm{K}, 0}$ generates the output response variation with energy. This response curve $\mathcal{R}_{\mathrm{MCP}}\left(\mathcal{E}_{\mathrm{K}, 0}\right)$ is then linearly fitted to scale to the experimental data points and allows retrieving the parameters $a$ and $b$ in Eq. (4). The result of this scaling (red full curve), together with the experimental values (black triangles), is presented in Fig. 2(c). We show the model for protons (red full curve) along with the experimental data points (black triangles) obtained through the pixel cluster analysis described above. It is possible to note that the response function reaches its maximum when the travel range of protons $R\left(\mathcal{E}_{\mathrm{K}, 0}\right)$ is approximately equal to $l_{\mathrm{g}}$, occurring at $\mathcal{E}_{\mathrm{K}, 0}=1.4 \mathrm{MeV}$. This particular initial energy maximizes the overlap between the exponential gain weight and the stopping power variation with depth $x$. For $R\left(\mathcal{E}_{\mathrm{K}, 0}\right)<l_{\mathrm{g}}$, the gain is high but a smaller amount of energy is deposited in the detector, explaining the continuously increasing response with increasing proton energy up to $1.4 \mathrm{MeV}$, in agreement with what is observed in the literature for $\mathrm{keV}$-ranged proton detection using MCPs. ${ }^{29,30}$ For $R\left(\mathcal{E}_{\mathrm{K}, 0}\right)>l_{\mathrm{g}}$, the response decreases since 
most of the particle's energy is deposited farther in the microchannel, where the gain is lower due to a shorter distance available for electron avalanche amplification. This effect is in agreement with previous works on $\mathrm{MeV}$-ranged protons. ${ }^{26,27,31}$ To retrieve the scaling factor for carbon ions, we use the same parameters $l_{\mathrm{g}}, a$, and $b$ as obtained for the best fit of protons. We then implement the calculations using the electronic stopping power of carbon ions in leaded-glass, as obtained from SRIM, to generate the scaling factor function for carbon ions, as shown in Fig. 2(d). As shown experimentally also in the work of Jeong et al., ${ }^{27}$ the response function for carbon ions is charge state-independent for this particular $\mathrm{MeV}$ range of energies since their Bragg peaks are positioned very close to the surface in the first few micrometers, hence making the response mostly energy-dependent. It is possible to observe in Fig. 2(d) that the model for carbon ions has the same shape as for protons but with its peak shifted at around $32 \mathrm{MeV}$ and also scaled up in intensity because the stopping power of carbon ions at the Bragg peak is much higher than for protons $(\sim 13 \times)$. We would like to stress that this methodology can be applied for other MCP detectors. In order to retrieve the response function, it is however necessary to measure at least three energy points, such as to define completely and univocally Eq. (4). To produce better results, it is advisable to use more data points, in particular, if the working energies are around the peak of the curve.

\section{THE ALLS 100 TW LASER-DRIVEN ION ACCELERATION BEAMLINE}

\section{A. Experimental setup}

We tested the absolute intensity calibration of our MCP-TP on the ALLS 100 TW ion acceleration beamline located at the Institut National de la Recherche Scientifique (INRS) in Varennes close to Montréal, Canada. The Ti:sapphire ALLS 100 TW laser $^{32}$ operates at a repetition rate of $2.5 \mathrm{~Hz}$ at a central wavelength of $\lambda_{0}=800 \mathrm{~nm}$ and comprises a double-Chirped Pulse Amplification (CPA) ${ }^{33}$ system delivering laser pulses with an energy on target of $\mathcal{E}_{L}=2 \mathrm{~J}$, in a pulse duration of $\tau_{L}=20 \mathrm{fs}$. Using an $f / 3$ off-axis parabola, the $100 \mathrm{~mm}$ beam size (at $e^{-2}$ ) is focused down to a spot size of $w_{\mathrm{FWHM}}=$ $5 \mu \mathrm{m}$, leading to a peak intensity of $I_{0} \sim 1.3 \times 10^{20} \mathrm{~W} / \mathrm{cm}^{2}$. The laser beamline benefits of a cross-wave polarizer (XPW) as part of a beam cleaning technique before injecting in the second CPA, which leads to an Amplified Spontaneous Emission (ASE) pre-pulse contrast of $<10^{-10}$ at -100 ps before the main pulse, along with a steep power rise with contrast of $<10^{-6}$ at $-3 \mathrm{ps}$. The p-polarized laser pulses are incident with an angle of $20^{\circ}$ with respect to target-normal on thin copper foils with a thickness of $5 \mu \mathrm{m}$ for performing laser-driven ion acceleration in the TNSA regime. The targets are mounted on a multi-target holder allowing for 16 shots per pumping cycle. The targets are first pre-aligned on a target alignment bench outside the interaction chamber and, subsequently, undergo micrometric precision alignment under vacuum using shadowgraphic imaging in the transverse plane, along with a target positioning interferometer. The accelerated ions are monitored by three particle diagnostics: one Thomson Parabola (TP) spectrometer placed at $0^{\circ}$ with respect to the target-normal axis along with two TOF diagnostic lines, one positioned at $+6^{\circ}$, and the other at $-9^{\circ}$. A schematic diagram of the setup is shown in Fig. 3(a). Concerning the geometrical layout of the

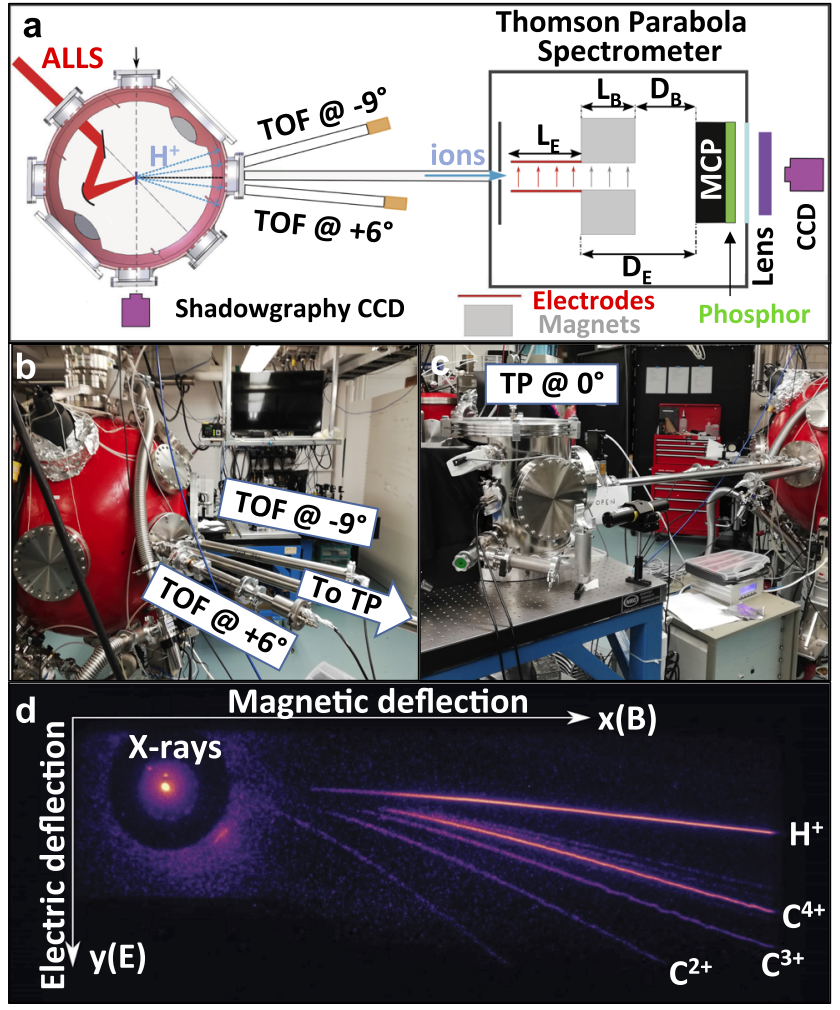

FIG. 3. (a) Experimental chamber layout and the TP spectrometer layout. (b) Picture of the experimental chamber and (c) a view from the rear side of the TP spectrometer experimental chamber. (d) A typical image obtained from the TP spectrometer. The other unlabeled parabola traces are the other carbon species that were not considered in the analysis of the present study due to their too low intensity as well as for better visualization purposes. In particular, the one on the left of $\mathrm{C}^{2+}$ is $\mathrm{C}^{+}$, along with the two above $\mathrm{C}^{4+}$ being $\mathrm{C}^{5+}$ and $\mathrm{C}^{6+}$.

TP shown in Fig. 3(a), the ions first enter through a $500 \mu \mathrm{m}$ pinhole placed $1.8 \mathrm{~m}$ away from the interaction point (i.e., collection solid angle of $6 \times 10^{-8} \mathrm{sr}$ ) before passing in between two $6 \mathrm{~cm}$-long copper electrodes that are separated by $2 \mathrm{~cm}$ and operated at $\pm 7.5 \mathrm{kV}$. The particles then go through a $0.46 \mathrm{~T}, 10 \mathrm{~cm}$-long magnetic field generated by permanent magnets, before spatially dispersing for $7.5 \mathrm{~cm}$, after which they are detected with the aforementioned MCP detection system that acquires a laser-triggered image of the different particle parabolas [see Fig. 3(d)]. The TOF lines are equipped with CVD-DDs. ${ }^{14}$ Both diamond detectors have an active layer of thickness $50 \mu \mathrm{m}$ grown on a commercial $4 \times 4 \times 0.5 \mathrm{~mm}^{3}$ High Pressure High Temperature (HPHT) substrate but present two different electrode layouts. The interdigit, or planar detector, placed at $-9^{\circ}$ has superficial interdigital aluminum contacts of $20 \mu \mathrm{m}$ width and 20 $\mu \mathrm{m}$ spacing. ${ }^{35,36}$ The sandwich, or transverse detector, placed at $+6^{\circ}$ has a layered structure consisting of a metal electrode deposited on the intrinsic diamond (i.e., the active volume of the detector), which, in turn, is in contact with a p-doped diamond. ${ }^{37}$ The two have different sensitivities for different energy ranges, and the planar diamond is more suitable for measurements of moderate-low energy protons and is characterized by a high temporal resolution due to the 
reduced distance between the electrodes. The transverse configuration is slightly slower but guarantees a quasi-uniform electric field throughout the whole thickness of the detector, providing a high Charge Collection Efficiency (CCE) for a wider energy range. Due to the different sensitivities for distinct energy ranges, the use of two types of diamond detectors ensured that we did not produce any systematic error in the estimation of the proton maximum energy nor of the proton yield. Since CVD-DDs are only sensitive to the energy deposited in their active layers, they are not able to distinguish different ion species. As we are mainly interested in retrieving proton spectra, both the detectors were supplied with a $10 \mu \mathrm{m}$ aluminum filter that provides a partial shielding from heavier ion contribution at the expense of loosing information on low-energy protons. From simulations performed using SRIM, it was possible to retrieve the maximum energies stopped by this filter, which are $750 \mathrm{keV}$ for protons, $11.45 \mathrm{MeV}$ for carbon ions, $13.7 \mathrm{MeV}$ for nitrogen ions, and $15.84 \mathrm{MeV}$ for oxygen ions, which are the main ion species accelerated during the interaction. Moreover, the filter provides a cutoff energy of $\simeq 45 \mathrm{MeV}$ for copper ions coming from the bulk target. To have an accurate estimation of the actual temporal response of the detectors, both detectors were calibrated by exposing them to 5.486 $\mathrm{MeV} \alpha$ particles produced by the radioactive decay of americium. The signal collected with the CVD-DD was sent to a Tektronix DPO 7104 scope ( $1 \mathrm{GHz}$ bandwidth and 5 Gsamples/s sampling rate) through $15 \mathrm{~m}$ long calibrated RG 223 cables. The transmission line was characterized by measuring its total $S_{21}$ scattering parameter using the Agilent E8364B network analyzer. The employment of long and highly shielded cables, together with the optimized shielding of the detectors itself, ${ }^{35,39,40}$ provided a good mitigation of the effects of EMPs that are generated during the laser-matter interaction. $^{41}$ The cables were sufficiently screened to avoid the electric field produced by the EMP from affecting the measurement. These EMPs, which can reach orders of hundreds of $\mathrm{kV} / \mathrm{m}$, can affect all the electronic devices placed nearby the experimental chamber. The proton spectra recorded with the diamond detectors located at $+6^{\circ}$ and $-9^{\circ}$ were computed following the procedure described by Salvadori et al., ${ }^{42}$ while the TP spectrometer allowed to simultaneously obtain the ion spectra at $0^{\circ}$. The particle number estimation retrieved from the parabolic traces shown in Fig. 3(d) was obtained with the pixel intensity calibration of the MCP detection system performed on the tandem accelerator at the Université de Montréal (see Sec. II B).

\section{B. Cross-calibration of the field integrals}

In order to properly analyze the images obtained on the MCP when used as a detector inside the TP spectrometer, it is necessary to follow correctly the traces of the trajectories belonging to the different ions traveling with different energies inside a magnetic and an electric field. The magnetic and electric fields might not be completely uniform during the particle's trajectory; it is therefore better to introduce two field integrals $I_{B}$ and $I_{E}$, defined by Eqs. (5) and (6). As shown in the work of Ducret et al., ${ }^{43}$ characterizing the field integrals allows us to bring a more general representation of the electric $\boldsymbol{E}$ and magnetic $\boldsymbol{B}$ fields used for TP spectrometers, avoiding the need to assume top-hat field distributions along the longitudinal axis. The values of $I_{B}$ and $I_{E}$ can further be inserted in the well-known non-relativistic, non-paraxial dispersion equations for the solution of charged-particle kinematics traversing static electric and magnetic fields for the case of Thomson parabolas, as shown by Eqs. (7) and (8),

$$
\begin{gathered}
I_{B}=\int_{0}^{L_{B}} \boldsymbol{B}(z) \mathrm{d} z=\overline{\boldsymbol{B}} L_{B}, \\
I_{E}=\int_{0}^{L_{E}} \boldsymbol{E}(z) \mathrm{d} z=\overline{\boldsymbol{E}} L_{E}, \\
x\left(\mathcal{E}_{\mathrm{K}}, I_{B}\right)=\frac{\sqrt{2 \mathcal{E}_{\mathrm{K}} m} L_{B}}{q I_{B}}\left\{\cos \left[\arcsin \left(\frac{q I_{B}}{\sqrt{2 \mathcal{E}_{\mathrm{K} m}}}\right)\right]-1\right\} \\
-D_{B} \tan \left[\arcsin \left(\frac{q I_{B}}{\sqrt{2 \mathcal{E}_{\mathrm{K}} m}}\right)\right], \\
y\left(\mathcal{E}_{\mathrm{K}}, I_{E}\right)=\frac{q I_{E}}{2 \mathcal{E}_{\mathrm{K}}}\left(\frac{L_{E}}{2}+D_{E}\right) .
\end{gathered}
$$

In Eqs. (7) and (8), $q, m$, and $\mathcal{E}_{\mathrm{K}}$ are the particle-dependent parameters, namely, its charge, mass, and kinetic energy, respectively. The field-related distances $L_{B}, D_{B}, L_{E}$, and $D_{E}$ are, respectively, the magnetic field length, the magnetic drift distance, the electric field length, and the electric drift distance. All of the aforementioned field-related distances are measured experimentally and hence are known values. Therefore, a proper calibration of the field integrals $I_{B}$ and $I_{E}$ allows us to completely define the system in terms of the particle's kinetic energy $\mathcal{E}_{\mathrm{K}}$ and charge-to-mass ratio $q / \mathrm{m}$. Using the results and formulas above, we have all tools at disposal to absolutely measure laser-accelerated protons in numbers and in kinetic energy using a MCP-TP system. In order to cross-calibrate the above-mentioned particle diagnostics, we adopted the following methodology:

(i) The magnetic field $\boldsymbol{B}(z)$ was measured with a calibrated gaussmeter. We used $L_{B}$ as a first approximation to determine $I_{B}$.

(ii) We move the TOF detector temporarily on the $0^{\circ}$ axis. Over a statistically significant amount of laser shots, we measure the maximum proton energy.

(iii) After placing back the TOF and the TP to their initial positions, we adjust $I_{B}$ until we get a good match between the two maximum energies obtained on the $0^{\circ}$ axis by the TOF and $\mathrm{TP}$. The maximum proton energy ratios at different angles (i.e., energy at $+6^{\circ}$ vs $0^{\circ}$ ) should be in agreement with typical TNSA-based proton beam values found in the literature. ${ }^{44,45}$

(iv) Both the voltage across the electrodes $\Delta V$ and the electrodes' separation distance $d$ including their length $L_{E}$ are measured and used as a first approximation to determine $I_{E}$.

(v) For a given ion species (protons, for instance), $I_{E}$ is incremented until best match is achieved with the theoretical curves from Eqs. (7) and (8) and the experimental positions on the detector.

(vi) As a final verification, the theoretical curves can be generated for other ion species (carbon ions, for instance) using (7) and (8) and should match with experimental positions on the detector. This confirms the correct parameters $I_{B}$ and $I_{E}$. 
One should note that the above methodology can be applied if there is at least one TOF line as a complementary particle diagnostics to the TP spectrometer. This particle diagnostic configuration allows using detectors located away from the $0^{\circ}$ axis to retrieve indirectly the characteristics of the beam on the central axis.

\section{Experimental results}

Since the TOF technique coupled with diamond detectors ensures an accurate measurement of the maximum proton energy, ${ }^{42,46}$ this technique was used to validate the kinetic energy estimations obtained with the TP spectrometer using the aforementioned cross-calibration methodology of the field integrals (see Sec. III B). In Fig. 4(a) is shown a comparison between the maximum proton energy estimated by the TP and the two TOF lines for different shots performed in similar conditions (i.e., using the same laser pulse energy and copper $5 \mu \mathrm{m}$ targets). The mean maximum energy of the selected shots is of $6.6 \pm 0.9 \mathrm{MeV}$ for the TP at $0^{\circ}, 5.9 \pm 0.8 \mathrm{MeV}$ for the TOF at $+6^{\circ}$, and $4.9 \pm 0.7 \mathrm{MeV}$ for the TOF at $-9^{\circ}$. It is possible to observe a good agreement between all three diagnostics that respond similarly for each shot. All shots exhibit small shot-to-shot fluctuations with a standard deviation on the maximum energy of $14 \%$ for the three diagnostics, indicating a good repeatability. In Fig. 4(b), the proton spectrum obtained with
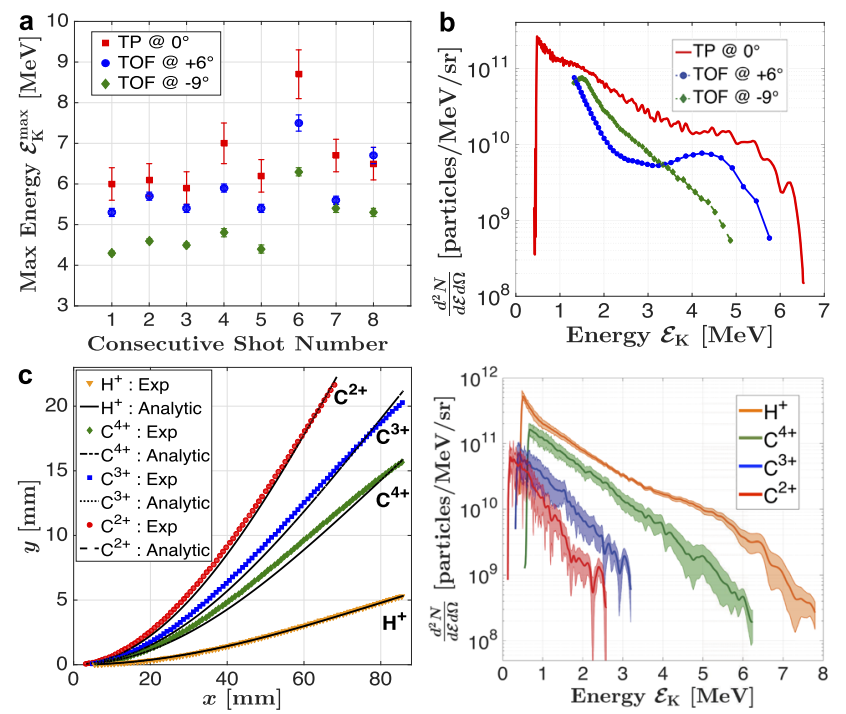

FIG. 4. (a) Maximum proton energies at different angles obtained by the three used diagnostics: The two TOF lines placed at $-9^{\circ}$ and $+6^{\circ}$, as well as the TP spectrometer placed at $0^{\circ}$. All the data are obtained with shots performed on 5 $\mu \mathrm{m}$ copper targets. Measurement was performed over 8 shots during which all diagnostics were operational. (b) Comparison among the spectra obtained by the aforementioned diagnostics for a typical shot. (c) Analytical parabolas (lines) calculated from Eqs. (7) and (8), along with experimental points (markers) obtained with physical distance measures on the MCP detection system. (d) Spectra obtained for different ion species $\left(\mathrm{H}^{+}\right.$in orange, $\mathrm{C}^{4+}$ in green, $\mathrm{C}^{3+}$ in blue, and $\mathrm{C}^{2+}$ in red) from the TP spectrometer. Each spectrum is an average of ten shots, and the uncertainties are calculated using the standard error of the mean. The low-energy cutoff corresponds to the lowest kinetic energy detectable for each ion species, and only particle numbers above the background floor are displayed. the TP spectrometer during a typical shot is shown together with the spectra provided by both TOF lines. The lower energy limit in the TOF spectra is determined by the growing tolerances due to the presence of the filter that produce higher uncertainty for energies close to the proton energy cutoff. For higher energies, two different ranges have to be taken into account. Up to $3 \mathrm{MeV}$, the behavior of the two diamond detectors is well characterized ${ }^{35}$ since protons are completely stopped inside the active thickness of the detector, depositing all their kinetic energy. Above $3 \mathrm{MeV}$, a correction factor has to be introduced to properly estimate the proton numbers. The uniform response of the diamond detector in the transverse configuration (i.e., $+6^{\circ}$ ) allows us to easily take this into account. ${ }^{46}$ On the other hand, the energy-dependent charge collection efficiency of the planar configuration (i.e., $\left.-9^{\circ}\right)^{42}$ does not allow us to follow the same procedure, and hence, a new methodology has to be implemented if a calibration with high-energy protons is not available, which will be the subject of a subsequent study. Consequently, the spectrum estimated with the TOF placed at $-9^{\circ}$ results to be underestimated for energies higher than $3 \mathrm{MeV}$ [green dashed line in Fig. 4(b)], whereas a reliable behavior is ensured for proton energies below 3 $\mathrm{MeV}$ [green full line in Fig. 4(b)]. Nevertheless, the spectra obtained with the TP spectrometer and both TOF lines exhibit a very typical TNSA-like shape, with the ratio of their cutoff energies in agreement with other reported TNSA divergences in the literature. ${ }^{44,45}$ Concerning Fig. 4(c), we note an excellent agreement between the analytical curves, generated using Eqs. (7) and (8) that were calibrated for their field integrals, and the experimental TP data points for different ion species as obtained by the MCP detection system. The matching is increasingly better with lower charge states, being best for protons, then for $\mathrm{C}^{2+}$, etc. The divergence from the analytical equations is due to the fringe fields at the extremities of the magnets and the electrodes, which bring higher-order field components to deflections. Another source of error comes from the fact that Eqs. (7) and (8) consider $\boldsymbol{E}$ and $\boldsymbol{B}$ fields that are invariant in the transverse plane, hence only varying along the longitudinal axis $z$. This changes the amplitude of the dispersion but is of negligible importance compared to the effects of the fringe fields since its effect can be incorporated within the calibration of the field integrals. Nevertheless, due to the relatively compact TP setup (i.e., small dispersion distances $D_{B}$ and $D_{E}$ ), the types of errors are minimized and the effect of the zero-order field components is dominant, as can be noted with the strong agreement for the proton parabola over a large magnetic deflection distance of $x=85 \mathrm{~mm}$. The very good agreement with the analytical equations along with the consistency observed within the three diagnostics validates the method we chose to crosscalibrate the TP spectrometer using the information retrieved by the two TOF lines.

In Fig. 4(d), we present the mean particle spectra for four ion species $\left(\mathrm{H}^{+}, \mathrm{C}^{4+}, \mathrm{C}^{3+}\right.$, and $\left.\mathrm{C}^{2+}\right)$, which are averaged over 10 shots and presented with their respective particle number uncertainty using the standard error of the mean. Similar to the observations regarding the maximum energy in Fig. 4(a), we can again note the high proton beam stability [orange curve in Fig. 4(d)] compared to similar laser-driven ion acceleration experiments, with a standard deviation of $15 \%$ for the amount of particles in its central section around $3 \mathrm{MeV}$. This is due to both the very small shot-to-shot fluctuations of the laser ( $2.5 \% \mathrm{rms}$ fluctuations in laser pulse energy) and the repeatable, high-precision $(<10 \mu \mathrm{m})$ alignment of the 
targets. The mean integrated proton number over the entire spectrum is in excess of $4.1 \times 10^{11}$ protons/sr as there is a substantial amount of undetected protons below the lower energy detection threshold of $0.4 \mathrm{MeV}$. The mean maximum energy is around 7.3 $\pm 0.5 \mathrm{MeV}$. Concerning the other presented ion species, the most favorably accelerated is $\mathrm{C}^{4+}$ for this laser energy value, as is clearly observed in Fig. 3(d) and Fig. 4(d). The mean integrated number of $\mathrm{C}^{4+}$ ions within the spectra is in excess of $1.7 \times 10^{11} \mathrm{particles} / \mathrm{sr}$, about $2.4 \times$ lower than protons, with a standard deviation in its central section of $55 \%$. The mean maximum energy for $\mathrm{C}^{4+}$ is around $6.2 \pm 0.4 \mathrm{MeV}$. The two other presented carbon ion species $\left(\mathrm{C}^{3+}\right.$ and $\mathrm{C}^{2+}$ ) appear in much smaller quantities, with a mean integrated number of particles in the first half of $10^{10}$ particles/sr, an order of magnitude lower than protons, with their maximum energies in the range of (2.2-3.2) MeV.

\section{DISCUSSION}

\section{A. Particle number scaling factor}

A proper characterization of the MCP's response is essential for the retrieval of the particle number scaling factor. By comparing the response function for $\mathrm{MeV}$-range ions obtained in this study and the one from the work of Jeong et al., ${ }^{27}$ we can first note a good agreement in terms of functional shape and also with regard to their peak positions. For instance, the modeling of Jeong et al. ${ }^{2}$ yields a peak of the response around $4 \mathrm{MeV}$ compared to our 1.4 $\mathrm{MeV}$ for protons and $24 \mathrm{MeV}$ compared to our $32 \mathrm{MeV}$ for carbon ions. Several factors explain these differences, and the dominant factors are the different MCP characteristics (pore diameter, channel length, and pitch) along with the use of a single MCP compared to the chevron MCP used in the present study. This yields different and very detector-specific responses. Moreover, their modeling includes an angular variation of the response to correct for the different particle incidences at different energies. Their absolute particle number calibration was obtained in situ with a TP spectrometer and CR-39 detectors, rather than on a particle accelerator as proposed here. The geometry of the presented setup allowed us to neglect the angular variation of the response function since the collection solid angle was negligibly small $(2.6 \mathrm{msr})$ to account for, as shown recently by the works of Fehre et al. ${ }^{47}$ and Blase et al. ${ }^{48}$ Nevertheless, the peak positions of the response functions for protons and carbons obtained in the present study are still in the same range of energies (few MeV) as in the work of Jeong et al. ${ }^{27}$ This can be explained by the fact that both MCPs come from the same company, and therefore, the global response is similar. Concerning the work of Harres et al., ${ }^{31}$ the presented response function follows the same trend as the one shown here; in particular, the function has a decreasing scaling factor with increasing proton energy above 2 $\mathrm{MeV}$. Moreover, the amplitude of the scaling factor, i.e., the number of counts per particle, is similar to ours since they also use a double MCP in the chevron configuration, yielding a similar output gain. The modeling presented in the work of Harres et al. ${ }^{3}$ considers the surface (i.e., $x=0 \mu \mathrm{m}$ ) electronic stopping power in Inconel for protons above $2 \mathrm{MeV}$ and hence does not include the variation of the stopping power with depth in the channel. This explains why there is no observation of a peak in their scaling factor function and also prevents to properly model carbon ions as is mentioned in their work. In light of these observations, it appears very important to properly model the response of an MCP detection system using the full stopping power dependence with depth (the full Bragg peak) in the correct material (leaded-glass here). As shown in several instances in the literature and again in the present study, approximating the depth-dependence of the MCP gain through a channel using an exponential behavior gives satisfying results.

Another important observation concerns the position of the peak at $1.4 \mathrm{MeV}$ displayed in Fig. 2(c) for the scaling factor of protons. By looking carefully at Fig. 3(d), it is possible to note on the proton parabola that there is a "high signal region" (hot spot) with more intense pixel intensities located around the middle of the parabola/detector. The position of this high signal region does not move from shot to shot, and since proton numbers decrease with increasing kinetic energy, it means that this effect is not related to particle numbers but rather to a higher response of the MCP at this particular proton energy. The proton kinetic energy related to this high signal region is about $1.5 \mathrm{MeV}$, which can also be noted as a small bump in the proton signal of Fig. 4(b) (red full line). The fact that the high signal region is located around the peak of the scaling factor function supports the correctness of our particle energy cross-calibration. Conversely, no high signal region is observed on the carbon parabolas of the MCP since the carbon kinetic energies generated during the acceleration process are lower than the peak position of its scaling factor function located around $32 \mathrm{MeV}$. In the energy range of the $0 \mathrm{MeV}-10 \mathrm{MeV}$, as observed in the spectra, the response function is still ramping up monotonously [see Fig. 2(d)].

\section{B. Performance of the beamline}

The mean proton number and the maximum kinetic energy obtained on our facility [Fig. 4(d)] are in agreement with what is found on other $100 \mathrm{TW}$-range laser facilities. ${ }^{49-54}$ These facilities report best proton numbers (typically obtained on the $0^{\circ}$ axis) in the range of $10^{11}$ protons/sr, along with maximum kinetic energies in the range of $3 \mathrm{MeV}-17 \mathrm{MeV}$, depending on the used target thickness and the use of pre-pulse contrast enhancement techniques, in agreement with the $4.1 \times 10^{11}$ protons $/ \mathrm{sr}$ and $7.3 \pm 0.5 \mathrm{MeV}$ reported here as the mean integral proton number and mean maximum kinetic energy, respectively. The mean ratios of maximum kinetic energies obtained at $+6^{\circ}$ and $-9^{\circ}$ with respect to $0^{\circ}$ are of $0.92 \pm 0.08$ and 0.75 \pm 0.08 , respectively. These values are well in agreement with experimental measurements of TNSA proton beams reported in the literature, ${ }^{44,45}$ although slightly lower by $6 \%$ and $12 \%$, respectively, for $+6^{\circ}$ and $-9^{\circ}$. This discrepancy is due to several factors, namely, the laser pulse energy, the pulse duration, the numerical aperture of the offaxis parabola used, and the target thickness, which will all together influence the divergence of the proton source. It is important to note that our laser configuration does not include a deformable mirror that could have further improved the performance of the beamline. Best shots reach nearly $10^{12}$ protons/sr and $9 \mathrm{MeV}$ on the $0^{\circ}$ axis with our $5 \mu \mathrm{m}$ thick targets. It is likely that thinner targets (e.g., 1 $\mu \mathrm{m}$-thick targets, not available during this experiment) might favor an enhanced proton yield and higher maximum energy. However, we note a high reliability of the beamline, providing a standard deviation of $15 \%$ both in proton numbers in the central energy section 
and maximum energy. In addition, having one TOF line on each side of the $0^{\circ}$ line allows us to determine after the shot if the target was tilted in the laser's plane of incidence. This allows us to easily discard bad shots or to improve the target alignment system, which further enhances the beam reliability. Moreover, having both TOF lines disposed at different angles allows us to have a better estimation of the beam divergence. All these criteria are extremely important when it comes to using laser-driven beamlines to reliably test numerous applications, such as radiation stress testing, ultrafast nanocrystal generation, ${ }^{56}$ radiation-induced morphological changes, ${ }^{57}$ and laser-PIXE. ${ }^{5,59}$ In particular, in the latter, the irradiation of a secondary target might block the diagnostic at $0^{\circ}$, but not the TOF lines at other angles. The testing of the aforementioned applications will be the subjects of subsequent studies on the beamline.

\section{CONCLUSION}

In summary, the presented study illustrates the most recent setup of the ALLS 100 TW laser-driven ion acceleration beamline that uses cross-calibrated TP-TOF detectors as particle diagnostics. The MCP used for particle detection in the TP spectrometer has been calibrated in intensity, on the $2 \times 6 \mathrm{MV}$ tandem linear accelerator at the Université de Montréal, using single proton impacts to retrieve the particle scaling factor that is used to determine and benchmark the particle numbers observed in the TP for protons and carbon ions. The experimental data points of the scaling factor were obtained by performing a pixel cluster analysis of the proton impacts on the MCP, and a semi-empirical model was fitted to the data to extrapolate the calibration to higher kinetic energies and also to extend it to other ion species. The methodology of this intensity calibration is universal and can be applied to other kinetic energies in the tens of $\mathrm{keV}$ to tens of $\mathrm{MeV}$ range, to different ion species, or to other types of MCP detectors, hence making the technique broadly applicable. In our findings, we highlight the importance of modeling the full depth-dependence for the stopping power (i.e., the Bragg peak), which allows us to properly model the non-linearities of the response function. Two TOF lines using diamond detectors, placed at $+6^{\circ}$ and $-9^{\circ}$ with respect to the target-normal axis, were used to determine the field integrals related to the electric and magnetic dispersions for the TP spectrometer. The two TOF lines allow us to verify the proton beam alignment on a shot-to-shot basis and to characterize the beam divergence. This opens the possibility to know the proton beam characteristics on shots where the $0^{\circ}$ axis of the TP spectrometer is blocked by a secondary target, but not the axes of the TOF lines, enabling diverse laser-driven proton beam applications that require reliable data. The used particle diagnostics are compatible with the development of a high-repetition rate targetry, as opposed to the use of radiochromic films or image plates, and are therefore a crucial step in the planned automation of the beamline. The testing of laser-driven proton beam applications using a very high number of shots to ensure good statistics will be the subject of subsequent studies.

\section{AUTHORS' CONTRIBUTIONS}

S.V. and M.S. contributed equally to this work.

\section{ACKNOWLEDGMENTS}

This work was supported by FRQNT (nouveaux chercheurs, Grant No. 174726, Equipe 2016-PR-189974), the National Sciences and Engineering Research Council of Canada (NSERC) (Discovery Grant No. RGPIN-2018-05772 and the Alexander Graham Bell Graduate Scholarship for S.V.), Compute Canada (Job: Grant No. pve-323-ac), and the Canada Foundation for Innovation (CFI). This study has also received financial support from the French National Research Agency (ANR) in the frame of "the Investments for the future” Programme IdEx Bordeaux (Grant No. ANR-10-IDEX-0302). The work of F.C. was carried out within the framework of the EUROfusion Consortium and has received funding from the Euratom research and training program 2014-2018 and 2019-2020 under Grant Agreement No. 633053. The authors wish to thank L. Godbout for maintaining the EN-1 Tandem accelerator, J.-C. Kieffer for the support, and F. Fillion-Gourdeau for the help during the experimental campaign.

\section{DATA AVAILABILITY}

The data that support the findings of this study are available from the corresponding author upon reasonable request.

\section{REFERENCES}

${ }^{1}$ M. C. Downer, R. Zgadzaj, A. Debus, U. Schramm, and M. C. Kaluza, "Diagnostics for plasma-based electron accelerators," Rev. Mod. Phys. 90, 035002 (2018).

${ }^{2}$ H. Daido, M. Nishiuchi, and A. S. Pirozhkov, "Review of laser-driven ion sources and their applications," Rep. Prog. Phys. 75, 056401 (2012).

${ }^{3}$ S. C. Wilks, A. B. Langdon, T. E. Cowan, M. Roth, M. Singh, S. Hatchett, M. H. Key, D. Pennington, A. MacKinnon, and R. A. Snavely, "Energetic proton generation in ultra-intense laser-solid interactions," Phys. Plasmas 8(2), 542 (2001).

${ }^{4} \mathrm{~J}$. Fuchs et al., "Comparison of laser ion acceleration from the front and rear surfaces of thin foils," Phys. Rev. Lett. 94, 045004 (2005).

${ }^{\mathbf{5}} \mathrm{J}$. Fuchs et al., "Comparative spectra and efficiencies of ions laser-accelerated forward from the front and rear surfaces of thin solid foils," Phys. Plasmas 14, 053105 (2007).

${ }^{6}$ A. Kemp, J. Fuchs, Y. Sentoku, V. Sotnikov, M. Bakeman, P. Antici, and T. Cowan, "Emittance growth mechanisms for laser-accelerated proton beams," Phys. Rev. E 75, 056401 (2007).

${ }^{7}$ Y. Gao et al., "An automated, $0.5 \mathrm{~Hz}$ nano-foil target positioning system for intense laser plasma experiments," High Power Laser Sci. Eng. 5, e12 (2017).

${ }^{8}$ F. Consoli et al., "Laser produced electromagnetic pulses: Generation, detection and mitigation," High Power Laser Sci. Eng. 8, e22 (2020).

${ }^{9}$ J. Metzkes, L. Karsch, S. D. Kraft, J. Pawelke, C. Richter, M. Schürer, M. Sobiella, N. Stiller, K. Zeil, and U. Schramm, "A scintillator-based online detector for the angularly resolved measurement of laser-accelerated proton spectra," Rev. Sci. Instrum. 83, 123301 (2012).

${ }^{10}$ A. Macchi, M. Borghesi, and M. Passoni, "Ion acceleration by superintense laser-plasma interaction,” Rev. Mod. Phys. 85, 2 (2013).

${ }^{11}$ A. Macchi, "A review of laser-plasma ion acceleration," arXiv:1712.06443 (2017).

${ }^{12}$ E. Woryna, P. Parys, J. Wołowski, and W. Mróz, "Corpuscular diagnostics and processing methods applied in investigations of laser-produced plasma as a source of highly ionized ions," Laser Part. Beams 14, 293 (1996).

${ }^{13}$ V. Scuderi et al., "Time of flight based diagnostics for high energy laser-driven ion beams," J. Instrum. 12, C03086 (2017). 
${ }^{14}$ M. Marinelli, E. Milani, A. Paoletti, A. Tucciarone, and G. Verona Rinati, "High-quality diamond grown by chemical-vapor deposition: Improved collection efficiency in $\alpha$-particle detection," Appl. Phys. Lett. 75, 20 (1999).

${ }^{15}$ S. Nakamura et al., "Real-time optimization of proton production by intense short-pulse laser with time-of-flight measurement," Jpn. J. Appl. Phys., Part 1 45(34), L913-L916 (2006).

${ }^{16}$ D. Haffa et al., "I-BEAT: Ultrasonic method for online measurement of the energy distribution of a single ion bunch," Sci. Rep. 9, 6714 (2019).

${ }^{17}$ APD 2 PS 97X79/32/25/8 I 40:1 NR P43, PHOTONIS USA, Inc., www.photonis.com.

${ }^{18}$ BFLY-PGE-13H2M-CS, FLIR Integrated Imaging Solutions, Inc., www.flir.com. ${ }^{19}$ M. Mayer, "SIMNRA user's guide," Report No. IPP 9/113, Max-Planck-Institut für Plasmaphysik, Garching, Germany, 1997.

${ }^{20}$ J. Baumann, R. Gnewkow, S. Staeck, V. Szwedowski-Rammert, C. Schlesiger, I. Mantouvalou, and B. Kanngießer, "Photon event evaluation for conventional pixelated detectors in energy-dispersive X-ray applications," J. Anal. At. Spectrom. 33, 2043 (2018)

${ }^{21}$ R. J. Beuhler and L. Friedman, "A model of secondary electron yields from atomic and polyatomic ion impacts on copper and tungsten surfaces based upon stopping-power calculations," J. Appl. Phys. 48, 3928 (1977).

${ }^{22}$ E. J. Sternglass, "Theory of secondary electron emission by high-speed ions," Phys. Rev. 108, 1 (1957).

${ }^{23}$ H. J. Frischkorn and K. O. Groeneveld, "Heavy ion induced electron emission from solid surfaces," Phys. Scr. T6, 89-93 (1983).

${ }^{24}$ P. H. Soltz, M. A. Furman, J.-L. Vay, A. W. Molvik, and R. H. Cohen, "Numerical simulation of the generation of secondary electrons in the High Current Experiment," Phys. Rev. Accel. Beams 6, 054701 (2003).

${ }^{25}$ P. Thieberger, A. L. Hanson, D. B. Steski, V. Zajic, S. Y. Zhang, and H. Ludewig, "Secondary-electron yields and their dependence on the angle of incidence on stainless-steel surfaces for three energetic ion beams," Phys. Rev. A 61, 042901 (2000).

${ }^{26} \mathrm{R}$. Prasad et al., "Calibration of Thomson parabola-MCP assembly for multi$\mathrm{MeV}$ ion spectroscopy,” Nucl. Instrum. Methods Phys. Res., Sect. A 623, 712-715 (2010).

${ }^{27}$ T. W. Jeong, P. K. Singh, C. Scullion, H. Ahmed, K. F. Kakolee, P. Hadjisolomou, A. Alejo, S. Kar, M. Borghesi, and S. Ter-Avetisyan, "Experimental evaluation of the response of micro-channel plate detector to ions with $10 \mathrm{~s}$ of $\mathrm{MeV}$ energies," Rev. Sci. Instrum. 87, 083301 (2016).

${ }^{28}$ J. F. Ziegler, M. D. Ziegler, and J. P. Biersack, "SRIM-The stopping and range of ions in matter (2010)," Nucl. Instrum. Methods Phys. Res., Sect. B 268, 1818-1823 (2010).

${ }^{29}$ J. L. Wiza, "Microchannel plate detectors," Nucl. Inst. Methods 162, 587-601 (1979).

${ }^{30}$ G. W. Fraser, "The ion detection efficiency of microchannel plates (MCPs)," Int. J. Mass Spectrom. 215, 13-30 (2002).

${ }^{31} \mathrm{~K}$. Harres et al., "Development and calibration of a Thomson parabola with microchannel plate for the detection of laser-accelerated $\mathrm{MeV}$ ions," Rev. Sci. Instrum. 79, 093306 (2008).

${ }^{32}$ S. Vallières, P. Puyuelo-Valdes, M. Salvadori, C. Bienvenue, S. Payeur, E. d'Humières, and P. Antici, "The laser-driven ion acceleration beamline on ALLS 200 TW for testing nanowires targets," Proc. SPIE 11037, 1103703-1 (2019).

${ }^{33}$ D. Strickland and G. Mourou, "Compression of amplified chirped optical pulses," Opt. Commun. 56, 219 (1985).

${ }^{34}$ C. Bienvenue, S. Vallières, S. Payeur, and P. Antici, "Submicrometric absolute positioning of flat reflective surfaces using Michelson interferometry," Rev. Sci. Instrum. 90, 123702 (2019).

${ }^{35} \mathrm{M}$. Marinelli et al., "Analysis of laser-generated plasma ionizing radiation by synthetic single crystal diamond detectors," Appl. Surf. Sci. 272, 104 (2013).

${ }^{36} \mathrm{~V}$. I. Polyakov et al., "Photodetectors with CVD diamond films: Electrical and photoelectrical properties photoconductive and photodiode structures," Diamond Relat. Mater. 7, 821 (1998).
${ }^{37}$ S. Almaviva et al., "Chemical vapor deposition diamond based multilayered radiation detector: Physical analysis of detection properties," J. Appl. Phys. 107, 014511 (2010).

${ }^{38} \mathrm{M}$. Cipriani et al., "Spectral characterization by CVD diamond detectors of energetic protons from high-repetition rate laser for aneutronic nuclear fusion experiments," J. Instrum. 14, C01027 (2019).

${ }^{39}$ R. D. Angelis et al., "High performance diagnostics for Time-Of-Flight and Xray measurements in laser produced plasmas based on fast diamond detectors," J. Instrum. 11, C12048 (2016).

${ }^{40} \mathrm{C}$. Verona et al., "Spectroscopic properties and radiation damage investigation of a diamond based Schottky diode for ion-beam therapy microdosimetry," J. Appl. Phys. 118, 184503 (2015).

${ }^{41} \mathrm{P}$. Bradford et al., "EMP control and characterization on high-power laser systems," High Power Laser Sci. Eng. 6, E21 (2018).

${ }^{42} \mathrm{M}$. Salvadori et al., "Accurate spectra for high energy ions by advanced time-offlight diamond-detector schemes in experiments with high energy and intensity lasers," arXiv:2003.01442 (2020).

${ }^{43}$ J.-E. Ducret et al., "Calibration of the low-energy channel Thomson parabola of the LMJ-PETAL diagnostic SEPAGE with protons and carbon ions," Rev. Sci. Instrum. 89, 023304 (2018).

${ }^{44} \mathrm{P}$. R. Bolton et al., "Instrumentation for diagnostics and control of laseraccelerated proton (ion) beams," Phys. Med. 30, 255-270 (2014).

${ }^{45} \mathrm{~A}$. Mancic et al., "Isochoric heating of solids by laser-accelerated protons: Experimental characterization and self-consistent hydrodynamic modeling," High Energy Density Phys. 6, 21 (2010).

${ }^{46} \mathrm{G}$. Milluzzo et al., "A new energy spectrum reconstruction method for timeof- flight diagnostics of high-energy laser-driven protons," Rev. Sci. Instrum. 90, 083303 (2019).

${ }^{47} \mathrm{~K}$. Fehre et al., "Absolute ion detection efficiencies of microchannel plates and funnel microchannel plates for multi-coincidence detection," Rev. Sci. Instrum. 89, 045112 (2018).

${ }^{48}$ R. C. Blase, R. R. Benke, G. P. Miller, K. S. Pickens, and J. H. Waite, "Microchannel plate detector detection efficiency to monoenergetic electrons between 3 and $28 \mathrm{keV}$," Rev. Sci. Instrum. 88, 053302 (2017).

${ }^{49} \mathrm{~K}$. Zeil et al., "The scaling of proton energies in ultrashort pulse laser plasma acceleration," New J. Phys. 12, 045015 (2010).

${ }^{50} \mathrm{O}$. Willi et al., "Particle and $\mathrm{x}$-ray generation by irradiation of gaseous and solid targets with a 100 TW laser pulse," Plas. Phys. Control. Fusion 51, 124049 (2009).

${ }^{51}$ O. Lundh, F. Lindau, A. Persson, C.-G. Wahlström, P. McKenna, and D. Batani, "Influence of shock waves on laser-driven proton acceleration," Phys. Rev. E 76, 026404 (2007).

${ }^{52}$ A. Pirozhkov et al., "Laser-driven proton acceleration and plasma diagnostics with J-KAREN laser," Proc. SPIE 7354, 735414-1 (2009).

${ }^{53} \mathrm{~S}$. Fourmaux et al., "Investigation of laser-driven proton acceleration using ultra-short, ultra-intense laser pulses," Phys. Plasmas 20, 013110 (2013).

${ }^{54} \mathrm{~J}$. Fuchs et al., "Laser-driven proton scaling laws and newpaths towards energy increase," Nat. Phys. 2, 48-54 (2006).

${ }^{55}$ M. Barberio, M. Scisciò, S. Vallières, F. Cardelli, S. N. Chen, G. Famulari, T. Gangolf, G. Revet, A. Schiavi, M. Senzacqua, and P. Antici, "Laser-accelerated particle beams for stress testing of materials," Nat. Commun. 9, 372 (2018).

${ }^{56}$ M. Barberio, M. Scisciò, S. Vallières, S. Veltri, A. Morabito, and P. Antici, "Laser-generated proton beams for high-precision ultra-fast crystal synthesis," Sci. Rep. 7, 12522 (2017).

${ }^{57}$ M. Barberio, S. Vallières, M. Scisciò, G. Kolhatkar, A. Ruediger, and P. Antici, "Graphitization of diamond by laser-accelerated proton beams," Carbon 139, 531-537 (2018).

${ }^{58}$ M. Barberio and P. Antici, "Laser-PIXE using laser-accelerated proton beams," Sci. Rep. 9, 6855 (2019).

${ }^{59}$ M. Barberio, S. Veltri, M. Scisciò, and P. Antici, "Laser-accelerated proton beams as diagnostics for cultural heritage,” Sci. Rep. 7, 40415 (2017). 